\title{
Exchanging personal health data with electronic health records: $A$ standardized information model for patient generated health data and observations of daily living
}

\author{
Panagiotis Plastiras \\ City University of London \\ Dympna O'Sullivan \\ Technological University Dublin, dympna.osullivan@tudublin.ie
}

Follow this and additional works at: https://arrow.tudublin.ie/scschcomart

Part of the Computer Sciences Commons

\section{Recommended Citation}

Panagiotis Plastiras, Dympna O'Sullivan, Exchanging personal health data with electronic health records: A standardized information model for patient generated health data and observations of daily living, International Journal of Medical Informatics, Volume 120, 2018, Pages 116-125, ISSN 1386-5056, DOI: 10.1016/j.ijmedinf.2018.10.006.

This Article is brought to you for free and open access by the School of Computer Sciences at ARROW@TU Dublin. It has been accepted for inclusion in Articles by an authorized administrator of ARROW@TU Dublin. For more information, please contact arrow.admin@tudublin.ie, aisling.coyne@tudublin.ie,gerard.connolly@tudublin.ie.

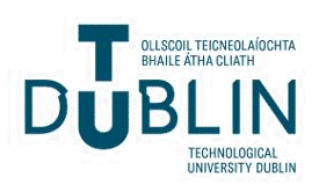




\title{
Exchanging personal health data with electronic health records: A standardized information model for patient generated health data and observations of daily living
}

\author{
Panagiotis Plastiras ${ }^{\mathrm{a}, *}$, Dympna O'Sullivan ${ }^{\mathrm{a}, \mathrm{b}}$ \\ ${ }^{a}$ Department of Computer Science, City University of London, Northampton Square, London EC1V 0HB, United Kingdom \\ ${ }^{\mathrm{b}}$ School of Computing, National College of Ireland, Mayor Street, North Dock, Dublin 1, D01 Y300, Ireland
}

\section{A R T I C L E I N F O}

\section{Keywords:}

HL7 CDA

Personal health record

Observations of daily living

Patient generated health data

Interoperability

\begin{abstract}
A B S T R A C T
Objective: The development of a middleware information model to facilitate better interoperability between Personal and Electronic Health Record systems in order to allow exchange of Patient Generated Health Data and Observations of Daily Leaving between patients and providers in order to encourage patient self-management.

Materials and methods: An information model based on HL7 standards for interoperability has been extended to support PGHD and ODL data types. The new information models uses HL7 CDA to represent data, is instantiated as a Protégé ontology and uses a set of mapping rules to transfer data between Personal and Electronic Health Record systems.

Results: The information model was evaluated by executing a set of use case scenarios containing data exported from three consumer health apps, transformed to CDA according to developed mapping rules and validated against a CDA schema. This allowed various challenges to emerge as well as revealed gaps in current standards in use and the information model has been refined accordingly.

Discussion and conclusion: Our proposed middleware solution offers a number of advantages. When modifications are made to either a Personal or Health Electronic Health Record system or any integrated consumer app, they can be incorporated by altering only the instantiation of the information model. Our proposition uses current standards in use such as CDA. The solution is applicable to any EHR system with HL7 CDA support.
\end{abstract}

\section{Introduction}

As healthcare technologies evolve, the management of health data is no longer only clinician-governed but also patient-controlled [1]. Engagement of the public with consumer health IT has been augmented by recent technological advances such as the use of mobile devices and the development of numerous health apps [2]. Until recently, Electronic Health Records (EHRs) were seen as the main vehicle to drive healthcare systems forward, however many researchers are now highlighting the vital role to be played by patients in controlling their own health information and self-managing their diseases [3,4]. These recent developments place the Personal Health Record (PHR) at the center of healthcare [5]. PHRs are health records that can be drawn from multiple sources and that are managed, shared, and controlled by the individual where these sources increasingly include consumer health applications and devices. Initiatives in this space are far reaching, for example, the Medicare and Medicaid EHR Incentive Programs in the US provide financial incentives for the "meaningful use" of health technology, which includes engaging and empowering patients and their families through the use and management of their health data in a PHR [6].

PHR adoption has historically been slow [7]. The main reasons highlighted by the literature are related to privacy and confidentiality, health literacy and integration and interoperability [8]. Other explanations include lack of consumer involvement in PHR design and development [9]; PHRs that provide access to clinical data from EHR which is not useful for patients [10]; and scant focus on individual tailoring, personalization and behavioral feedback which are important aspects of personal health management [11]. However as the scope of PHRs increases to include consumer health applications and devices, adoption rates are increasing rapidly, yet scant support exists for exchanging personal health data from the range of PHRs with EHRs.

There are two prevailing models of PHR systems. The first are "untethered" standalone systems which are entirely under the control

\footnotetext{
* Corresponding author.

E-mail address: Panagiotis.Plastiras.1@city.ac.uk (P. Plastiras).
} 
of the patient who must enter their own information or arrange for it to be transferred from another system. This group includes consumer health applications and devices. The second are "tethered" systems, often referred to as patient portals, which are sponsored by an organization and where the record is automatically populated with institutional clinical data such as hospital visits or prescription drugs dispensed without the patient needing to enter information. The vast majority of existing and emerging PHR systems are untethered; therefore the success of these systems is determined by a person's willingness to maintain their PHR information or on their Health Care Provider's (HCP) willingness to share and transfer data to an EHR. The tethered model places less burdens on the patient, however few are designed to allow patients to upload their own information and so are not compatible with consumer applications and devices and the adaption of these systems to allow sharing of institutional and personal data usually presents costly and time-consuming challenges for HCPs [12-14]. Moreover, a recent systematic review [14], reported significant issues in the use of 47 out of 60 tethered PHRs reviewed, including interoperability issues, a lack of support for user needs, and in particular, a lack of functionality to allow users to define what data to integrate was identified as a major barrier.

In previous work, we proposed an information model to enable better interoperability between PHR and EHR systems, where concepts, relationships, rules and constraints were derived by analyzing widely used PHR features and functionality [15]. The information model was inspired by the HL7 Reference Information Model (RIM) [reference] of the health care information domain and is designed as a middle layer between PHR and EHR systems. It composed an ontology that modelled concepts and relationships, and a set of mapping rules that outlined rules and constraints that must be followed when integrating data from a PHR data with an EHR and vice versa. Data can be exchanged between systems using common standards such as XML and the Clinical Document Architecture (CDA) [16].

In this paper, we extend the information model by updating it to facilitate newly identified needs of PHR such the integration of patient generated health data (PGHD) and observations of daily living (ODL). PGHD and ODLs are health-related data generated by patients and gathered via consumer health apps, sensors and devices. They reflect the extended use of PHRs to incorporate not only health-related data but also other data pertaining to general wellbeing, diet and fitness used by both healthy and unhealthy persons to monitor their health and lifestyle. PGHD and ODLs are mainly defined by individuals and are meaningful to them and/or their caregivers. They can be interpreted to provide insights into someone's health and wellbeing and to assist to self-care and self-management including setting goals and changing health behaviors [11]. Widely used PGHD include chronic diseases measurements such as blood glucose levels [17], [18], whereas ODLs include measurements such as activity and nutrition [11].

In this paper, we outline the development of the information model extension to facilitate PGHD and ODL data exchange between PHR and EHR. We describe the required changes to the information model, the underlying ontology and to the mapping rules to facilitate the new data types. We demonstrate how data from three mobile health apps is captured and transformed to CDA format ready for insertion in an EHR via the information model.

\section{Background}

A PHR has been defined by the Markle Foundation as "an electronic application through which individuals can access, manage and share their health information, and that of others for whom they are authorized, in a private, secure and confidential environment" [19]. Similarly Archer et al [20] define PHRs as "Internet-based tools that allow people to access and coordinate their lifelong health information and make appropriate parts of it available to those who need it". It has been noted that a universal definition has not yet been agreed to describe
PHRs [8].

Studies including [21-23] list basic PHR functionality as storage of and access to personal information, past and current medications, vital signs, orders and laboratory results and procedures. Sophisticated requirements such as the capture of PGHD and ODLs from consumer health devices are not reflected in such lists. Initiatives such as Meaningful Use [6] have brought PHRs firmly into focus by emphasizing patient engagement using patient portals and enabling patient integration with provider EHRs. A lack of efficient interoperability remains one of the main barriers for PHR adoption [7,14,24]. Slow adoption of standards such as the Continuity of Care Record (CCR) and the Continuity of Care Document (CCD) has delayed the rollout of PHR systems. The use of existing standards such as SNOMED CT can facilitate semantic interoperability however a uniform PHR standard that supports the same communications, messaging, and content encoding standards, in particular encoding lay representation of data, as other health information systems has not yet been reached [25].

Further challenges to interoperable PHR are the fact that ideal information in a PHR is lifelong and cross institutional, and thus should cover data from various HCPS as well as citizens own entries and emerging data from apps, sensors and integrated devices [7,14]. A related issue is how to allow individuals to specify what own data they wish to be shared with HCPs and associated systems. Further, citizens are the primary managers of the data but may also want to share it with other non-clinical stakeholders, for example family members [26]. As such, authentication presents a problem for PHRs. Another complicating factor is that increasingly dispersed care across HCPS and EHRs is the general rule given the increasing frequency of co-morbid chronic conditions [27]. This poses major challenges due to possible resultant provider-to provider misunderstandings, information gaps, and identification of responsibility.

Recent reports and work have highlighted specific barriers to the incorporation of PGHD and ODLs in PHRs. The Healthcare Information and Management Systems Society (HIMSS) describe information overload, complications in trend analysis and meaningful presentation as pertinent issues relating to PGHD and ODL data. They also point to limitations in technology standards and technology reliability relating to apps, sensors and devices from which PGHD and ODL data must be transferred and exchanged with PHRs [28].

Project HealthDesign is a multi-year, multi-site project that supports a wide range of patients, from children with chronic health conditions to elders transitioning from hospital to home [29]. It allows capture of medical data and ODLs and uses a third party PHR, namely Microsoft HealthVault as a secure data repository. This allows access for patients and clinicians as well as information exchange between both parties. One of the main challenges faced during the project was the fact that existing data models do not incorporate new essential data types such as ODLs [30]. The researchers report that the cost of designing and developing sophisticated interfaces to facilitate complex data types soon outweighed the expected benefits [8].

Sujansky and Kunz [29] proposed a model based on DIRECT messaging secure email standards [31,32], for collection of patient data using personal health devices and the secure sharing of these data with authorized providers' EHRs. They reported that a significant drawback of their approach is that data defined by individuals such as PGHDs do not directly map to biomedical models of disease and illness and are also are more likely to be sent in an unstructured way.

However the rise of smartphones, sensors and devices powered by Internet of Things and mobile health apps to create and record PGHD and ODLs have the potential to move healthcare in the direction of true patient-centeredness [33,34]. PGHD and ODLs allow for effective selfcare and self-management via individual tailoring, personalized reporting and behavioral feedback [5]. For example, by combining demographics, individual routines and behavioral habits available via smart devices, personalized interventions or treatments can be designed based on individual characteristics. These can be supplemented with 


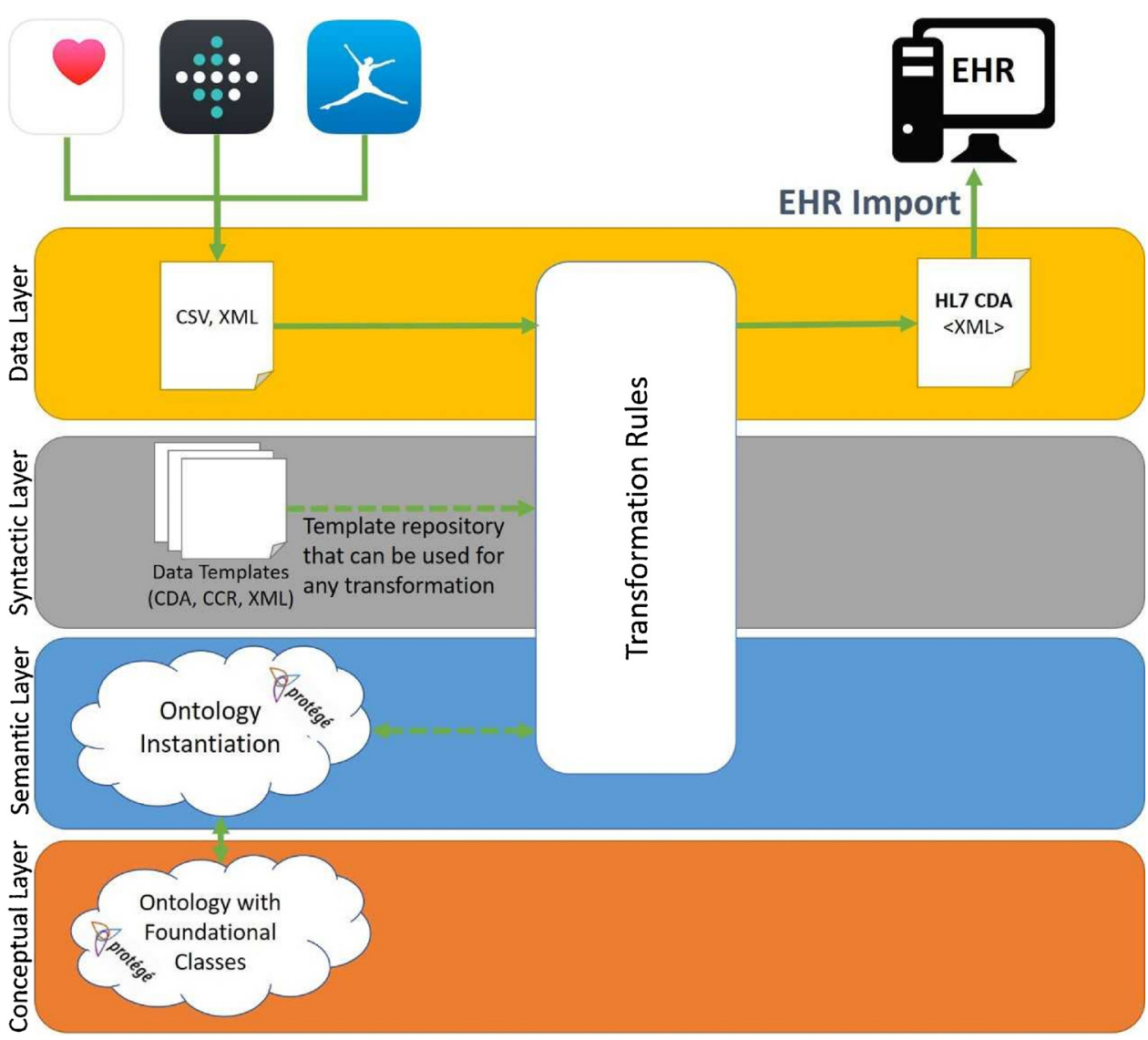

Fig. 1. Information model architecture outlining the workflow of exchanging data from a PHR with an EHR.

behavioral feedback or advice from clinicians and be used to encourage persons to adhere to healthy behaviors or to take up or continue with interventions and treatments [11].

\section{Methods}

In previous work we developed an information model by analysing and modelling common features and functionality of PHRs [15]. In this work, we outline an extended information model that can incorporate PGHD and ODLs to allow for self-care and self-management by recording and monitoring such data and setting personal health goals, and methods for the transfer of such new data from PHRs to EHRs. Fig. 1 outlines the architecture of our information model and outlines the workflow of exchanging data between PHRs and EHRs. The architecture is composed of four structural components. The first component is the conceptual layer, namely the definition of each concept in the PHR and how concepts are related. The second component is the semantic layer which provides a formal representation of the entities defined in the conceptual layer and allows data to be mapped to standardized vocabularies SNOMED and LOINC. The ontology instantiation reflects the data and associated attributes a given application (e.g. Fitbit) can hold. The ontology is referenced from both the conceptual layer and the semantic layer to ensure the exported data are understood correctly using a vocabulary such as SNOMED and transformed to the appropriate format ready for import.

The third component is the syntactic layer which aims to ensure that represented data will fully conform to pre-defined structure and syntactic rules as outlined by standards such as HL7 CDA. Finally, the data layer manipulates data exported from either a PHR or EHR and creates a document that can be transferred between systems. The transformation rule engine implements the transformation rules to manipulate the data and create the document for transfer and lies between the data, the semantic and the syntactic layer. The specific PHRs outlined in Fig. 1 are three consumer health applications, Apple Health, Fitbit and MyFitnessPal as represented by their app icons. Note that our proposition assumes data is available for exchange with an EHR and does not include methods to export data from a PHR into our information model. This is because the code required to pull data from a PHR is bespoke for each app and device and would be best facilitated via a public API associated with the specific application or device.

In the next sections we briefly describe our original information model, we outline how we extend the information model to incorporate PGHD and ODLs and present an evaluation of the extended information model via case studies using personal health and wellness data transferred from three consumer health apps.

\subsection{Original information model}

We developed the original information model by analysing common PHR data and functionality [15]. The information model consisted of four fundamental classes, which were inspired by classes from the HL7 RIM standard:

- $\underline{\text { Role: }}$ an acknowledged and defined participant in one's PHR, e.g. a person in the Role of a patient or practitioner can participate in a PHR encounter with a set of defined permission on the Role, for example, a patient may be permitted to record health data in a PHR whereas a practitioner may not be allowed to add data, rather they can provide feedback on data recorded by the patient.

- Entity: each Role is played by an Entity, for example specific (named) patients and practitioners in a PHR are Entities.

- Act: an Act is any event in a PHR, for example, the Act of recording an allergy or monitoring blood pressure. An Entity participates in an Act, for example a named patient can record an allergy in a PHR. 


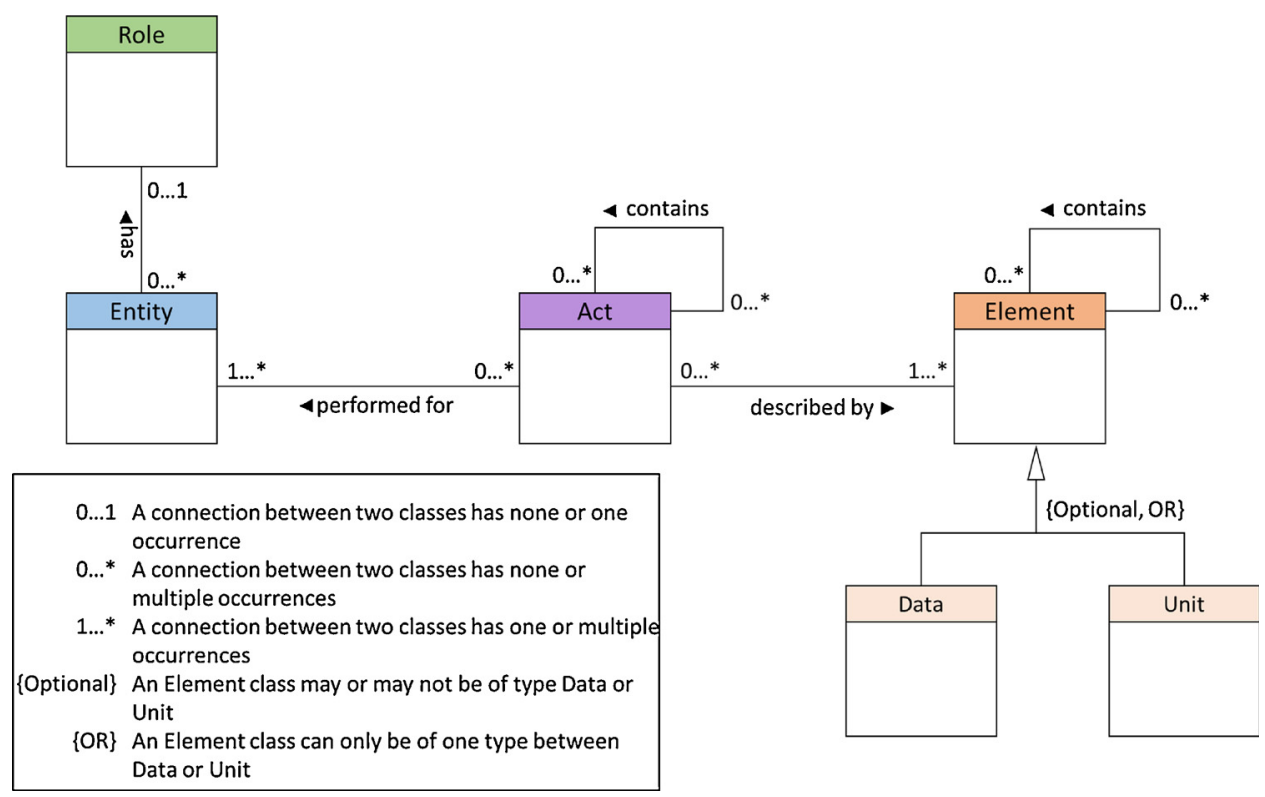

Fig. 2. Original information model.

- Element: an Element is any data corresponding to an Act, for example, an allergy recorded in a PHR is an Element. Elements may contain sub-elements, for example, allergy name and effective date. Moreover, the class Element has two subclasses named Data and Unit for recording fine grained Element data, for example an activity tracker may record a user's 10,000 -day step goal as Data $(10,000)$ and Unit (steps).

A UML representation of the original information model is shown in Fig. 2 below.

The Role and Entity classes from our information model are mapped directly to the respective RIM classes. We extend the functionality of the RIM Act class, in particular the way it interacts with the class Role. Roles in PHR systems (e.g. patient, care giver) are more limited than in EHR systems (e.g. access role, employee, licensed entity etc) and Entities in PHR systems participate directly in Acts. HL7 RIM does not have an Element class so the Element class from our information model is replicated utilizing the Observation class from RIM where an observation often involves measurements or other methods of investigation. Further, in contrast with RIM, the attributes of each class and the classes themselves are flexible so that the four classes can accommodate all relevant information from PHR systems. For complete details of the development of the information model using RIM classes please see our previous published work in [16].

The information model uses the classes defined above and their attributes to define how data is captured and subsequently how it is manipulated by receiving systems. An example of using attributes to define how data is manipulated is the property 'hasEHRTransferable' which is used to dictate whether an element should be transferred to an EHR or not. Similarly attributes can have attached properties such as 'hasSnomedCode' or 'hasLoincCode' to facilitate semantic interoperability by indicating data that has corresponding concept representation in a relevant biomedical terminology. This feature allows accommodation of synonymous data from multiple sources including applications and devices. For example both Fitbit and Apple Watch devices track sleep quality and duration, however their internal labeling of this data on the respective devices may be different. The information model allows Apple Watch and Fitbit data to be mapped to standardized SNOMED concepts 'Duration of sleep' and 'Quality of sleep'. While our information model can accommodate any data from a PHR, we acknowledge that standardized representation of such data is limited to the vocabulary defined by SMONED and LOINC. To date, attributes have been defined for use by Element objects to allow manipulation of data, however, they may be also used for other classes. For instance, in a situation where a user may want to restrict data transfer from specific devices, perhaps a device that they only use occasionally, a similar attribute could be defined for the Entity class.

The information model is underpinned by an ontology created using Protégé [35]. This ontology is used as a reference that outlines what data can be captured and whether the data should be processed and subsequently transferred between a PHR and an EHR [16]. In addition, a set of transformation rules for manipulating data exported from PHRs in order to transform it into a CDA document or XML file commonly used by EHRs have been developed.

The main advantage of the information model is that it is flexible enough to cover any kind of data used in a PHR context despite the heterogeneous nature of existing PHRs. We strive to provide a standardized representation of heterogeneous data as far as possible by allowing data to be described using equivalent SNOMED and LOINC codes. The middle layer architecture between a PHR and an EHR ensures flexibility by not tying the solution to any specific PHR or EHR system; when modifications are made to data or formats in a PHR or EHR system, their transformations can be incorporated directly by altering only the instantiation of the information model in the middle layer. The RIM inspired architecture emphasizes the exchange of full medical records rather than simple message exchange which allows for greater volumes of data to be transferred. Moreover, by developing an extensible information model based on the RIM class and attribute structure we can facilitate emerging PHR and EHR needs for new data types such as PGHD and ODLs.

\subsection{Developing the extended information model}

In order to extend the original information model to incorporate PGHD and ODLs, three widely-used consumer apps have been selected and analyzed in terms of the data they capture and their functionality. Inclusion was based on simple criteria including their popularity and the main functionality they provide is capture and storage of a wide variety of PGHD and ODLs. The selected apps are:

1 Fitbit [36], a smart device emphasizing fitness and wellbeing monitoring with an associated software app. The device 
automatically records activity data such as number of steps taken, and sleep times and the user may also self-report other activity and lifestyle data. The user can track data and view longitudinal data via an app on a smartphone or tablet. They can also set activity and weight loss goals. Fitbit may be integrated with other devices such as smart watches and scales as well as other third party apps.

2 Apple Health app [37], is available on iOS devices and helps to track numerous parameters including activity, nutrition, sleep data, vital signs and reproductive health data. It also supports integration with smart watches and other devices and the ability to receive and push data from and to numerous third party apps.

3 MyFitnessPal [38], is an app for monitoring nutrition; however, other body parameters such as height, weight and exercise can also be recorded. Nutrition data can be added to the app using an integrated food database, manually by the user or using barcode scanning functionality. The primary purpose of the app is to encourage weight loss so the user may set a number of goals in that regard. The MyFitnessPal app can be integrated with other devices including as smart watches and third party apps.

\subsection{Extending the information model classes}

One of the main features of consumer apps is that unlike traditional PHR which relied on manual data entry, they allow data to be recorded using either wearable technology such as smart watches or other electronic devices including activity monitors, scales, blood pressure monitors and alternative input methods such as barcode scanners. This allows effortless data recording for the end user and provides a more objective and reliable source since the accuracy of the data is only dependent on the accuracy of the device. In addition, continuous data can be recorded as soon as the wearable device is working and has battery, which allows data to be captured for entire days and provides a holistic view of a person's health or wellness state. Therefore, in our extended information model, the Role class needs to be extended to include such new Entities. Roles must now include any authorized devices and third party apps and corresponding Entities are specific named devices or apps (e.g. Fitbit, Apple Watch or MyFitnessPal).

As previously stated, many consumer apps allow users to set up goals and work towards achieving them. These goals can be auto generated from the app itself following a basic algorithm based on users' activities and responses or added and amended manually by users. In our extended information model the most suitable place to record such goal data is the Element class. In order to maintain a differentiation from Data and Unit elements a new sub-element named Goal it is proposed. In terms of HL7 RIM, the Goal Element subclass is an Observation subclass in the same manner as Data and Unit classes (see [16] for a full description of the information model in terms of RIM classes), but used to specifically store data about a user's goals. The extended Information Model can be seen in Fig. 3.

\subsection{Extending the ontology}

Updating the information model also requires updating the underlying ontology to reflect the new sub-element Goal. In addition, the ontology from the original information model represented the viewpoint of a single system (i.e. a single PHR). According to a recent study, approximately $65 \%$ of people using health related apps are using more than one app to track data and data from many apps is frequently combined [39]. As such the need to combine data from multiple sources emerges. As a result, the original ontology needed to be re-engineered to reflect a user-specific instantiation rather than a single PHR viewpoint, which can contain elements from more than one source system. For example, sleep pattern data can be tracked using more than one application (a Fitbit and an Apple Watch); however, the user might only want to integrate data captured by one of them or to integrate only the latest values regardless of the source application.

\subsection{Updating the mapping rules for transforming PHR-EHR and EHR-PHR} data

In our previous work [16] a set of mapping rules were proposed to facilitate smooth mapping of data extracted from a PHR and transform them into an HL7 CDA document ready to be integrated to an EHR and vice versa. In the extended information model, the transformation rules have been amended to facilitate the user-specific ontology instantiation rather than a single PHR viewpoint used in the original model. Previously, one of the rules checked to find the specific instantiation of the ontology that matched the exported app since there was one instantiation per app. In the extended information model there is one instantiation for all apps (single viewpoint), so it is no longer necessary to find the source app and choose the appropriate instantiation to check what data are expected.

\section{Evaluation of the extended information model}

We have selected varied pieces of PGHD and ODL data from the three consumer apps (Fitbit, Apple Health and My Fitness Pal) to demonstrate and evaluate our extended information model. Data has been exported from Fitbit, the Apple Health and MyFitnessPal apps to our information model in CSV or XML formats and transformed to HL7 CDA using the set of mapping rules. The data is as follows:

1 Sleep data from Fitbit;

2 Reproductive health aspects including menstruation from Apple Health;

3 Nutrition data from My Fitness Pal.

\subsection{Representing PGHD and ODL data in the extended information model}

Sleep data as recorded by a Fitbit device is represented in the extended information model as shown in Fig. 4. Note that the colour used for each element, matches the colour used for each class from the information model (Fig. 3). The Role is that of Fitbit since that device records the data. The Entity is the specific Fitbit device defined by the user. This device participates in the Act of monitoring sleep. The main Element 'sleep pattern' can be further broken down into further subelements such as time, end time, number of awakenings etc. Those subelements can be finally broken down to Data (total amount of sleep), Unit (minutes) and Goal (sleep goal in minutes as defined by the user) sub-elements. The circled numbers in Fig. 4 show how corresponding data from the Fitbit application is represented in the information model.

Considering the Apple Health app, users can either upload their data manually or the data can be automatically pulled through from other compatible third party apps and stored within Apple Health. Fig. 5 outlines a scenario where data regarding menstruation is recorded on another app (e.g. the Cycles app [40]), then transferred, and stored within Apple Health app. In this case the third party app is the main Role, Cycles is the specific Entity, monitoring is the performed Act and menstrual period flow is the main Element which can be broken down to more granular sub-elements such as menstruation, data, and start of cycle. As before, the circled numbers show how corresponding data from the Apple Health application is represented in the information model.

In a similar manner, nutrition data stored in the MyFitnessPal app can be represented as shown in Fig. 6. The app user is the main Role, the Entity is the specific person using the app, monitoring nutrition data is the performed Act and Element is broken down to contain a number of relevant sub-elements. Fig. 6 also demonstrates how a Goal subelement can be used to set a target number of calories. 


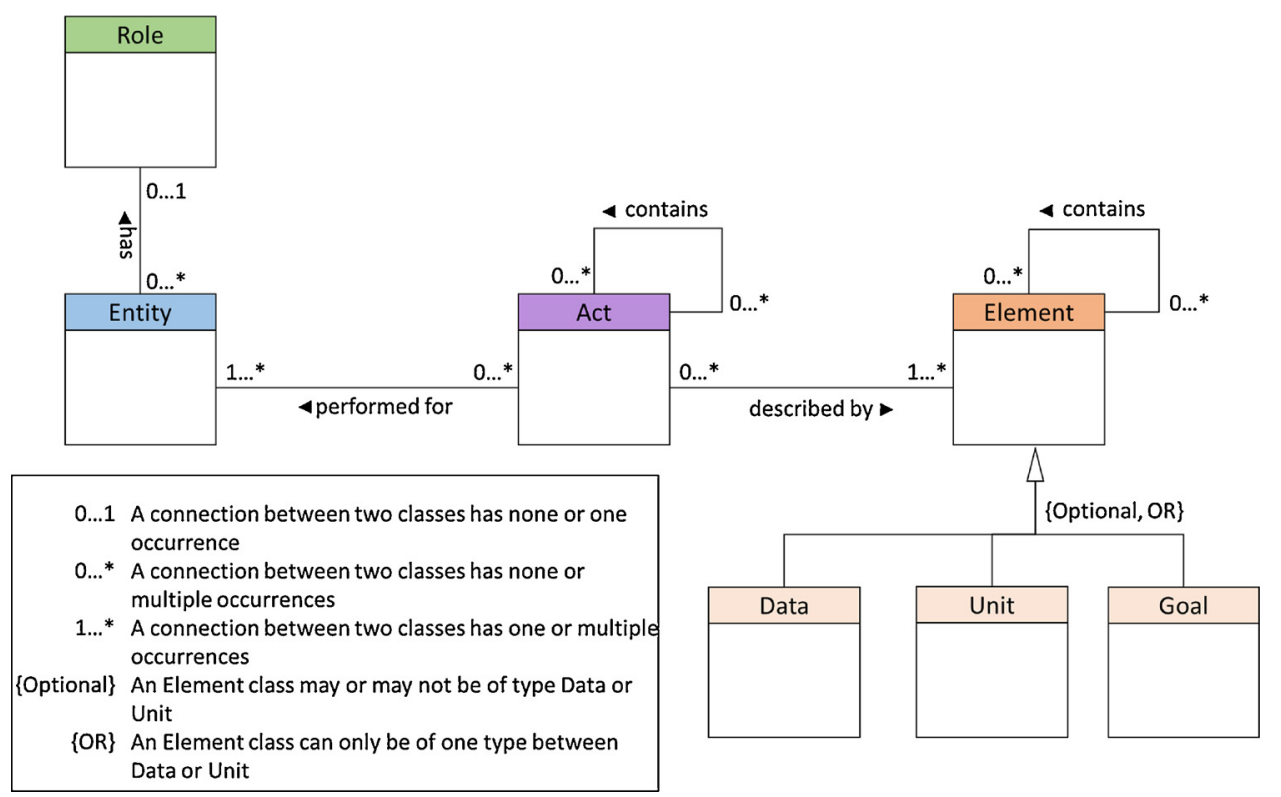

Fig. 3. Extended information model to include goals.

\subsection{Updating the ontology to facilitate PGHD and ODLs}

The ontology in the extended information model is user specific in order to achieve optimal data aggregation from multiple sources. Therefore, for the purposes of the evaluation we assume that a single user is using all three example apps in order to track data and hence we will instantiate a single user specific ontology to include all data from these apps. The instantiation can be expanded to cover any data used by any app connected to our information model. Thus, if a new app is added in the future and the patient is willing to integrate data from that app, the instantiation must be lengthened to include all the necessary data from the new app. Fig. 7 illustrates an example of the Element sleep pattern and the connections with other sub-elements in the ontology. Fig. 7 also shows how the information model can reconcile terminology from multiple source applications. The image shows how Fitbit describes sleep pattern data (see object property assertions) and how we map sleep data to a standardized vocabulary using LOINC code 28341-6 with description "Sleep And Rest Pattern Behavior" to store sleep pattern data. Other applications may label sleep pattern differently. By specifying a 'Same Individual as' relation which is one where two individuals are named differently, but refer to one and the same instance, we can define which data are conceptually equivalent even if they are derived from different applications (e.g. Fitbit's 'sleep pattern' versus Apple Health's 'sleep analysis'). Thus, we attempt to facilitate semantic interoperability between multiple source applications although we recognize that we cannot predict all terms and data that will be used by different manufacturers of devices and application and thus this is not a complete solution.

\subsection{Data transformation}

Our proposition has been evaluated by executing three scenarios in which sample data was exported from each application above (Fitbit, MyFitnessPal and Apple Health) and transformed to a CDA document according to the mapping rules outlined in [16]. The process is as follows:

The first step is to identify the participating Entities and Roles from the exported data. For all scenarios the required information was available within the instantiated ontology and was identified by parsing the ontology instantiation OWL file. In the next step the rule aims to

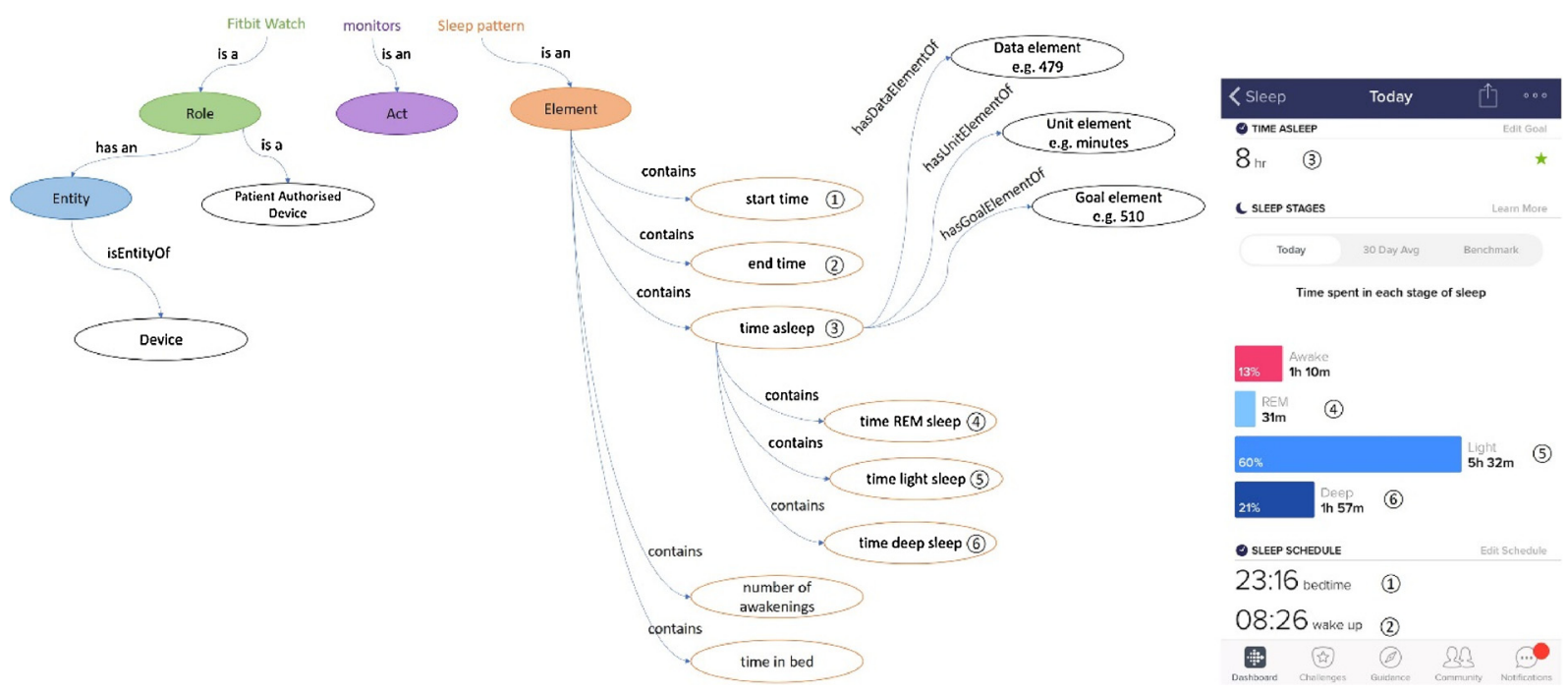

Fig. 4. Sleep pattern from Fitbit represented according to the extended information model. 


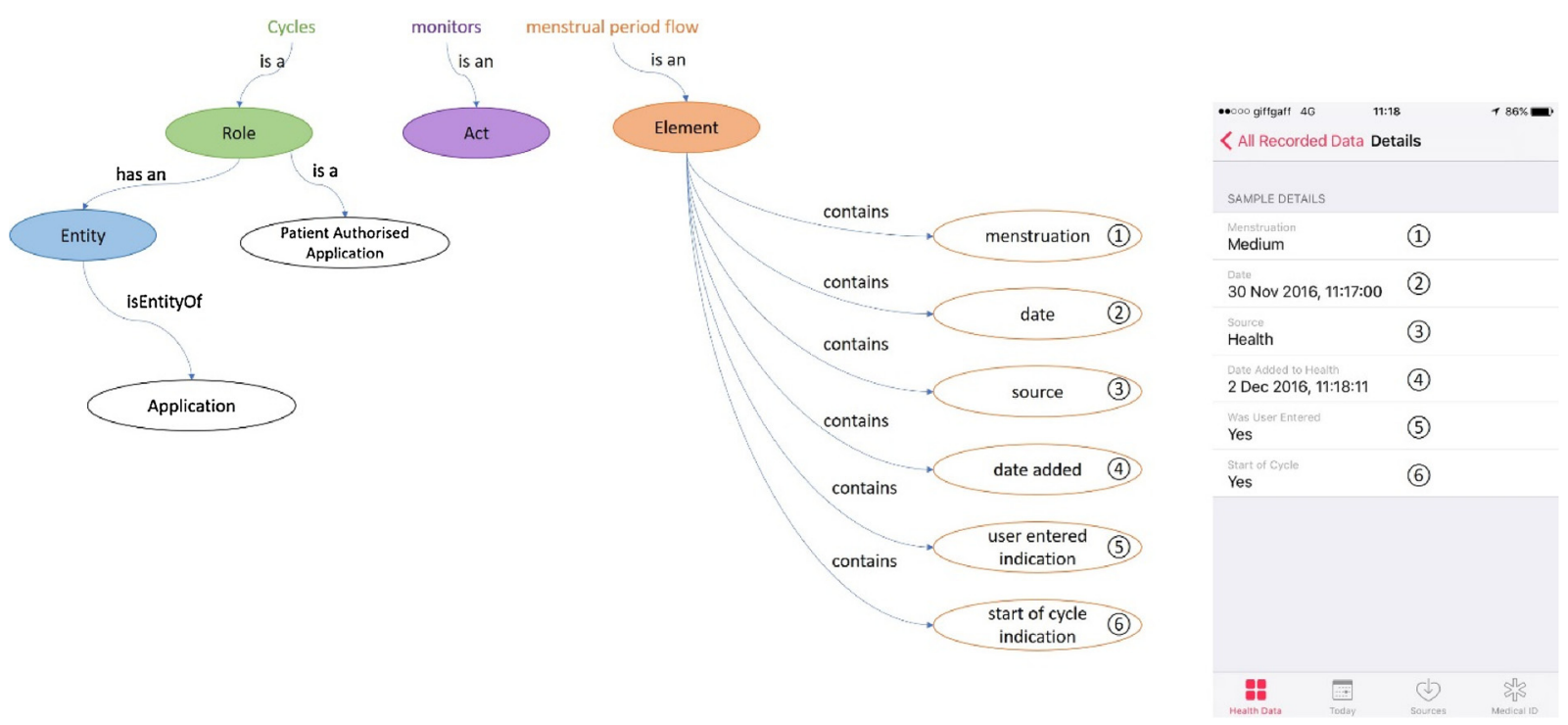

Fig. 5. Menstruation data imported to Apple Health via Cycles app represented according to the extended information model.

identify the different high-level Elements that have been exported. In the scenarios this corresponds to sleep pattern, menstrual period and nutrition details. In the third step, the ontology instantiation recognises which attributes for each of the Elements identified from the previous step should be expected from each application and the values for those attributes must be extracted from the exported data. Based on the preasserted attributes for each element, the algorithm decides how to proceed when processing each element, specifically whether the elements must be transferred to an EHR or not. Once the above has been established, pre-defined XML templates are used to allow the extracted data to be transferred to a CDA document. Finally, the last step is to construct the header based on data extracted from the first step and attach the header on top of the CDA document.

\subsection{Challenges encountered when executing the scenarios}

The first challenge faced was the increased scope of different Roles and Entities comparing to our previous work [16] where Person and Patient were used respectively. Considering the Fitbit scenario for monitoring sleep pattern data, the Role of the device is recorded as 'Patient Authorised Device' and the Entity has been recorded as the specific 'Device'. The device type (Fitbit Alta) was recorded as an attribute of the device Entity. This can be seen in Fig. 8 below.

The Fitbit has been assigned as a Patient Authorised Device role on the basis that when this information is integrated into a health record, clinicians must be able to differentiate between authorized or unauthorized devices. Other potential Roles, for example a patient measuring blood glucose levels using an unauthorised device (e.g. one
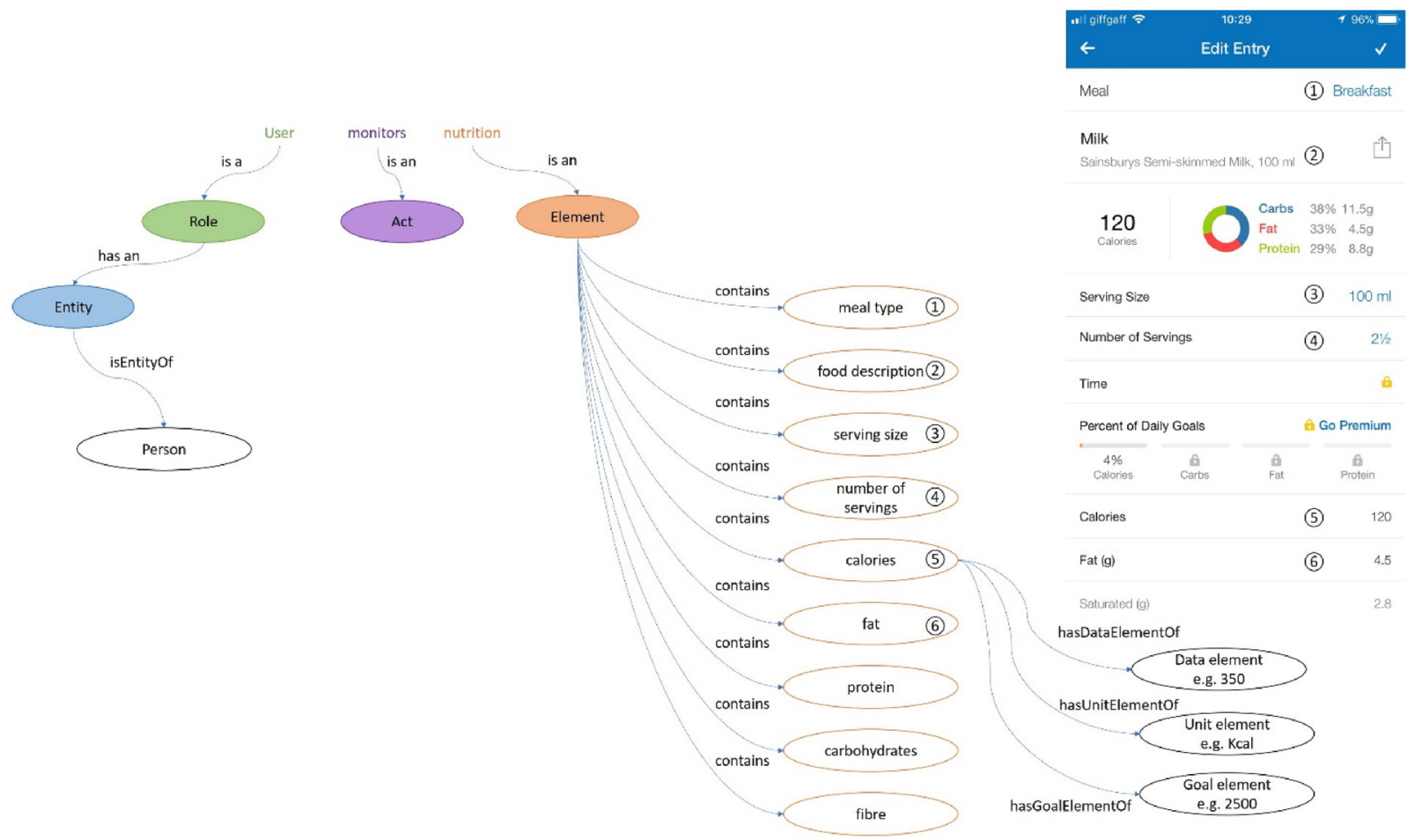

Fig. 6. Nutrition data recorded via MyFitnessPal app represented according to the extended information model. 


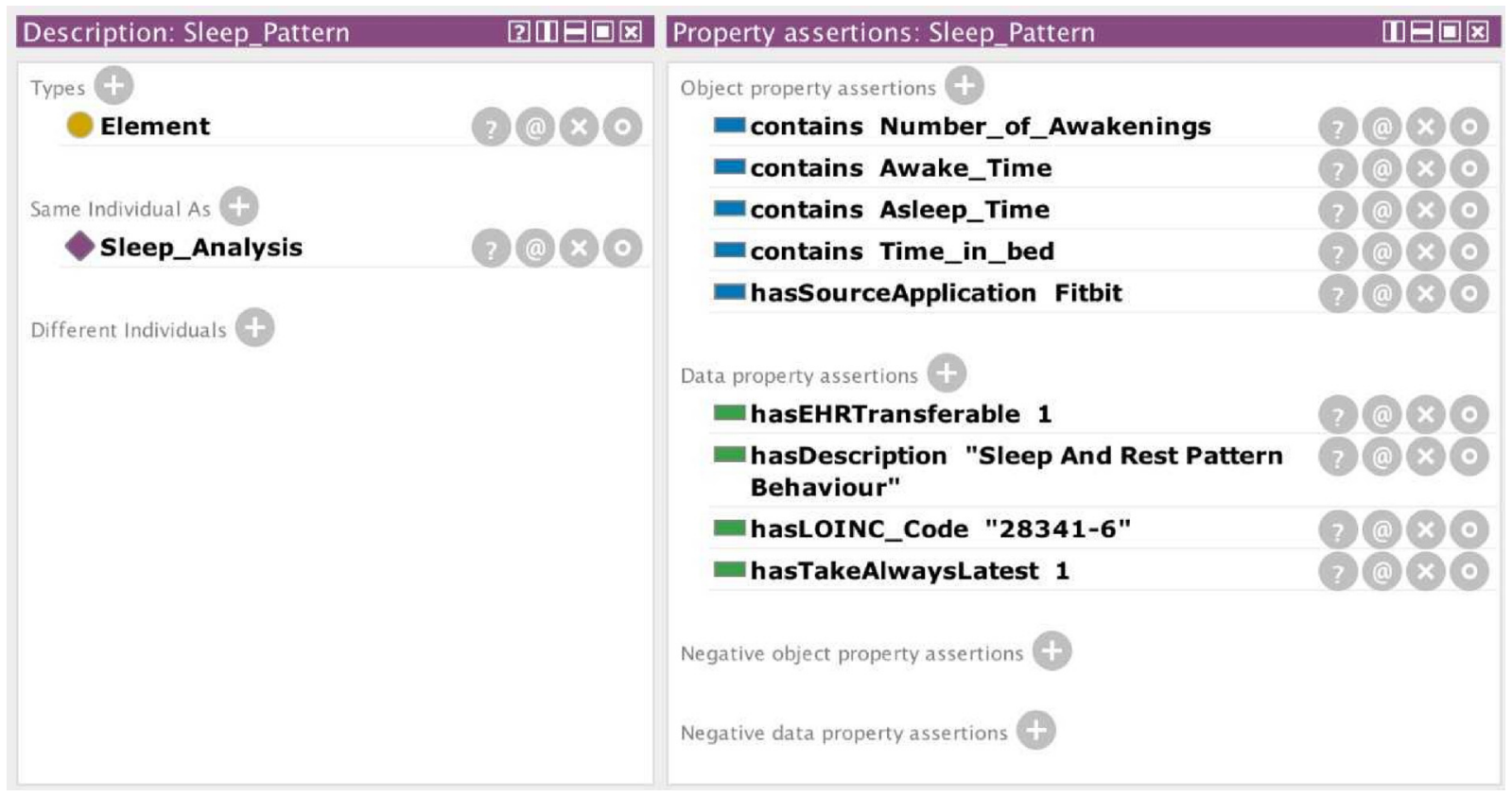

Fig. 7. Sleep Pattern Element with property assertions.

belonging to someone else), can be recorded as a 'Patient Unauthorised Device'. This will allow to detect and interpret small fluctuations to measurements due to different devices. In addition, it is possible to assign any data related to a 'Patient Unauthorised Device' by attaching a property 'EHRTransferable' with value $=0$, meaning that these values will not be communicated to an EHR and only stored in the PHR.

In the Apple Health scenario menstrual period data was automatically pulled through a compatible third-party app and not directly recorded on the Apple Health application. To address this situation, an Entity of 'Application' describing the third-party application with role of 'Patient Authorized Application' were used.

An additional challenge was the heterogeneous nature and thus the wider semantic interpretation of the exported PGHD and ODL data. Different standardized vocabularies were used in order to overcome this issue. For instance, the 'sleep pattern' Element was represented by the LOINC code '28341-6' and LOINC description 'Sleep and Rest Pattern
Behaviour' has been used. This can be seen in Fig. 9a below. However, sub-elements of 'sleep pattern' such as 'asleep time' could not be represented by LOINC codes and hence a SNOMED code of ' $248,220,008$ 'has been used (Fig. 9b). The coding schema varied based on the data item as no single schema supported all the exported data. For the purpose of this study, an attempt to map all the exported data to at least one terminology has been made, even if that means that the main highlevel element would be mapped to a different terminology from its subelements. This challenge could not be overcome due to the limitations coding schemas widely used have.

After the construction of the CDA document in XML format finished, the final document was validated into two different online CDA validators [41,42], successfully.

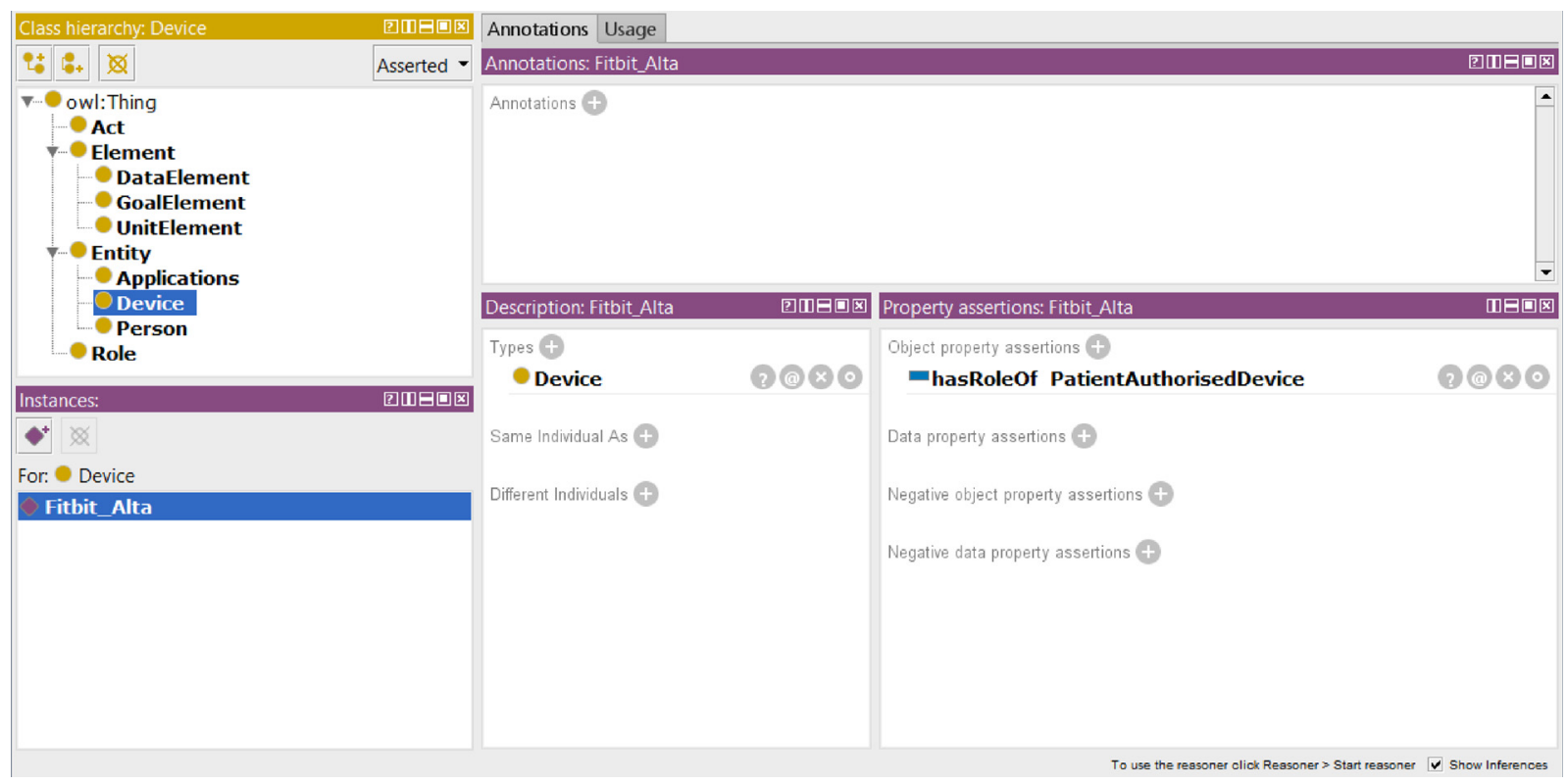

Fig. 8. Device Entity and link to the Role 'Patient Authorized Device'. 


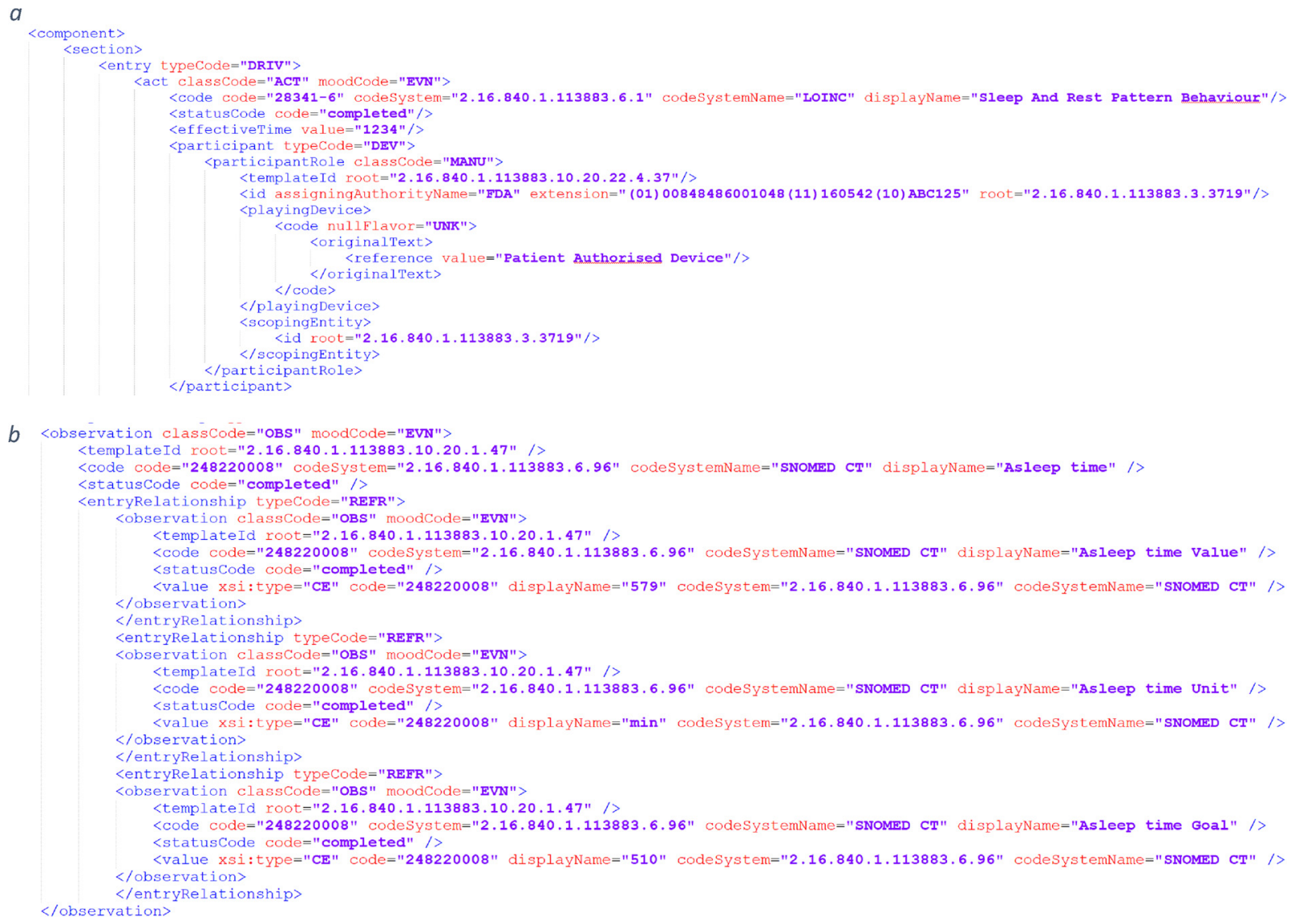

Fig. 9. a -Sleep and rest pattern behavior represented in CDA. b - Observation to express Asleep Time containing sub-elements for Data, Unit and Goal.

\section{Discussion and conclusion}

In this paper we have presented an extension to a previously developed information model [16] to allow for the exchange of personal health data such as PGHD and ODLs from mobile health apps with an EHR. The extended model also allows for the associated goals setting capabilities that often accompany such data. To reflect the fact that many consumer health apps and devices are used in conjunction with other third party devices as and apps the ontology underpinning the extended information model has been expanded to reflect a user-centric point of view allowing the integration of data from multiple source apps.

Our proposition was evaluated with data from three consumer applications. PGHD and ODL data has been captured and represented using our extended information model and transformed and exported to CDA format successfully.

In future work we intended to investigate how captured PHR data, represented by our information model can be exported using the HL7 FHIR (Fast Healthcare Interoperability Resources) standard. FHIR is optimized for mobile devices and apps and for simultaneously accessing data from different healthcare systems. FHIR uses building blocks called resources to describe data formats and elements and we intend to investigate how well PHR data including PGHD and ODL can be described using resources.

We also intend to investigate which PGHD and ODLs are most useful from a clinician's perspective and how those data should be represented in an EHR to provide a view of health status relevant to a clinician. Finally, we are considering the development of disease specific instantiation of our information model. For example, it would be interesting to develop an app for a long-term disease (e.g. diabetes) that requires regular monitoring of more than one parameter (e.g. nutrition, exercise, medication, blood sugar levels and pregnancy specific parameters) and sharing of selected data with a number of clinicians for shared decision making.

\section{Authors contributions}

All authors were involved in all aspects and have approved the manuscript for submission.

Study conception and design: Plastiras, O’Sullivan

Acquisition of data: Plastiras, O'Sullivan

Analysis and interpretation of data: Plastiras, O'Sullivan

Drafting of manuscript: Plastiras, O'Sullivan

Critical revision: Plastiras, O'Sullivan

Approval of the version of the manuscript to be published: Plastiras, O'Sullivan.

\section{Conflict of interest}

The authors whose names are listed immediately below certify that they have NO affiliations with or involvement in any organization or entity with any financial interest (such as honoraria; educational grants; participation in speakers' bureaus; membership, employment, consultancies, stock ownership, or other equity interest; and expert testimony or patent-licensing arrangements), or non-financial interest (such as personal or professional relationships, affiliations, knowledge or beliefs) in the subject matter or materials discussed in this manuscript.

\section{Summary points}

- We have expanded a previously developed information model to allow for the incorporation of more complex data such as 
observations of daily living and patient generated health data from consumer mobile applications and the associated goals setting capabilities that often accompany such data.

- We have adopted the use of Clinical Document Architecture (CDA) as an already established and widely used standard.

- Our proposition was evaluated using a set of case studies. Data from three different consumer applications has been exported and transformed to CDA. Subsequently, the data were validated successfully.

- One of the main disadvantages of CDA is the limited number of use cases it was initially designed to address and the fact that it cannot fully support current data types such as observations of daily living and patient generated health data.

\section{References}

[1] J. Wasson, H. Forsberg, S. Lindblad, G. Mazowita, K. McQuillen, E. Nelson, The medium is the (health) measure: patient engagement using personal technologies, J. Ambulatory Care Manag. 35 (Apr-Jun) (2012) 109-117.

[2] H. Greenspun, S. Coughlin, mHealth in an mWorld - how mobile technology is transforming healthcare, Deloitte Cent. Heal. Solut. (2012) 1-21.

[3] C. Fernandez-Llatas, A. Martinez-Romero, A.M. Bianchi, J. Henriques, P. Carvalho, V. Traver, Challenges in personalized systems for Personal Health Care, 3rd IEEE EMBS Int. Conf. Biomed. Heal. Informatics 2016 (2016) 356-359.

[4] T. Bodenheimer, K. Lorig, H. Holman, K. Grumbach, Patient self-management of chronic disease in primary care, JAMA 288 (19) (2002) 2469-2475.

[5] M.S. Househ, E.M. Borycki, W.M. Rohrer, A.W. Kushniruk, Developing a framework for meaningful use of personal health records (PHRs), Heal. Policy Technol. 3 (4) (2014) 272-280.

[6] "Meaningful Use Definition \& Objectives," HealthIT.gov, 2015.

[7] J.D. D'Amore, et al., Are Meaningful Use Stage 2 certified EHRs ready for interoperability? Findings from the SMART C-CDA Collaborative, J. Am. Med. Inform. Assoc. (2014) 1060-1068.

[8] J. Studeny, A. Coustasse, Personal health records: is rapid adoption hindering interoperability? Perspect. Health Inf. Manag. 11 (2014) 1e.

[9] S. Nundy, C.-Y.E. Lu, P. Hogan, A. Mishra, M.E. Peek, Using patient-generated health data from mobile technologies for diabetes self-management support: provider perspectives from an academic medical center, J. Diabetes Sci. Technol. 8 (1) (2014) 74-82.

[10] A.S.Y. David Dorr, Laura M. Bonner, Amy N. Cohen, Rebecca S. Shoai, Ruth Perrin, Edmund Chaney, Informatics systems to promote improved care for chronic illness: a literature review, J. Am. Med. Inform. Assoc. (2005) 156-163.

[11] U. Backonja, K. Kim, G.R. Casper, T. Patton, E. Ramly, P.F. Brennan, Observations of daily living: putting the 'personal' in personal health records, Nurs. informatics ... Proc. ... Int. Congr. Nurs. Informatics 2012 (2012) 6.

[12] L. Beard, R. Schein, D. Morra, K. Wilson, J. Keelan, The challenges in making electronic health records accessible to patients, J. Am. Med. Inform. Assoc. 19 (1) (2011) 116-120.

[13] F. Mold, et al., Patients' online access to their electronic health records and linked online services: a systematic review in primary care, Br. J. Gen. Pract. 65 (632) (2015) e141-e151.

[14] M.J. Thompson, J.D. Reilly, R.S. Valdez, Work system barriers to patient, provider, and caregiver use of personal health records: a systematic review, Appl. Ergon. 54 (2016) 218-242.

[15] P. Plastiras, D.O. Sullivan, P. Weller, An Ontology-Driven Information Model for Interoperability of Personal and Electronic Health Records, eTELEMED, 2014, pp. 130-133 no. c.
[16] P. Plastiras, D. O'Sullivan, Combining ontologies and open standards to derive a middle layer information model for interoperability of personal and electronic health records, J. Med. Syst. 41 (12) (2017) 195, https://doi.org/10.1007/s10916 017-0838-9 Oct 28

[17] S. Gupta, N. Kaur, D.K. Jain, mSwasthya: a mobile-enabled personal health record management system, Int. Conf. Comput. Commun. Autom. (2015) 374-379.

[18] G. C. Lamprinakos et al., "Using FHIR to develop a healthcare mobile application," Proc. 2014 4th Int. Conf. Wirel. Mob. Commun. Healthc. - "Transforming Healthc. Through Innov. Mob. Wirel. Technol. MOBIHEALTH 2014, pp. 132-135, 2015.

[19] Connecting for Health, "Overview and Principles," Common Framew. Networked Pers. Heal. Inf., 2008.

[20] N. Archer, U. Fevrier-Thomas, C. Lokker, K. a McKibbon, S.E. Straus, Personal health records: a scoping review, J. Am. Med. Inform. Assoc. 18 (4) (2014) 515-522.

[21] P. Francek, I. Piljic, L. Dragic, H. Mlinanc, M. Kovac, D. Gvozdanovic, Overcoming E-health interoperability obstacles: integrating PHR and EHR using HL7 CCD, Proc. Elmar - Int. Symp. Electron. Mar. 2015 November (September) (2015) 73-76.

[22] Y.-T. Song, J. Pak, A. Kalabins, and S. Fouché, "Standard-based Patient-centered Personal Health Record System".

[23] D.J. Cohen, S.R. Keller, G.R. Hayes, D.A. Dorr, J.S. Ash, D.F. Sittig, Developing a model for understanding patient collection of observations of daily living: a qualitative meta-synthesis of the Project HealthDesign program, Pers. Ubiquitous Comput. 19 (1) (2015) 91-102.

[24] C. Showell, "Barriers to the use of personal health records by patients: a structured review".

[25] D. Detmer, M. Bloomrosen, B. Raymond, P. Tang, Integrated personal health records: transformative tools for consumer-centric care, Med. Inform. 8 (1) (2008) 45.

[26] F. Wartena, J. Muskens, L. Schmitt, Continua: the impact of a personal telehealth ecosystem, Proc. - Int. Conf. eHealth, Telemedicine, Soc. Med. eTELEMED 2009, (2009), pp. 13-18.

[27] H.H. Pham, D. Schrag, A.S. O'Malley, B. Wu, P.B. Bach, Care patterns in Medicare and their implications for pay for performance, N. Engl. J. Med. 356 (11) (2007) 1130-1139.

[28] HIMSS, National eHealth Collaborative Technical Expert Panel Final Report, (2013).

[29] W. Sujansky, D. Kunz, A standard-based model for the sharing of patient-generated health information with electronic health records, Pers. Ubiquitous Comput. 19 (1) (2015) 9-25.

[30] P. Healthdesign, Project HealthDesign - Phase II : Grantee Technical Architectures and Implementations on Behalf of," October, (2011).

[31] “The Direct Project (2010) The Direct Project Overview." [Online]. Available: http://wiki.directproject.org/file/view/DirectProjectOverview.pdf. [Accessed: 22Jan-2018].

[32] "No TitleOffice of the National Coordinator for Health Information Technology (2013) What is the Direct Project?" [Online]. Available: http://www.healthit.gov/ providers-professionals/faqs/what-direct-project. [Accessed: 22-Jan-2018].

[33] A.B. Wilcox, K.D. Gallagher, B. Boden-Albala, S.R. Bakken, Research data collection methods: From paper to tablet computers, Med. Care 50 (2012).

[34] S. Fox, M. Duggan, Pew research center's internet and american life project, Mob. Heal. 2012 (2012).

[35] "Protégé." [Online]. Available: https://protege.stanford.edu/.

[36] "fitbit." [Online]. Available: https://www.fitbit.com/.

[37] Apple, "Apple Health App." [Online]. Available: https://www.apple.com/uk/ios/ health/.

[38] MyFitnessPal, "MyFitnessPal.".

[39] P. Krebs, D.T. Duncan, Health app use among US mobile phone owners: a national survey, JMIR Mhealth Uhealth 3 (4) (2015) e101.

[40] "Cycles - Period Tracker." [Online]. Available: http://perigee.se/apps/cycles/. [Accessed: 22-Jan-2018].

[41] "CDA Validator." [Online]. Available: https://www.lantanagroup.com/validator/ Validator. [Accessed: 28-Dec-2017].

[42] "CDA Guideline Validator." [Online]. Available: http://cda-validation.nist.gov/ cda-validation/validation.html. 